\title{
Tipologie di contaminazione nella tradizione testuale della "Commedia" dantesca
}

\author{
Elisabetta Tonello
}

\begin{abstract}
This essay examines the phenomenology of contamination in the textual tradition of Dante's Commedia. After clarifying the definition of contamination, and its relationship with the editio variorum, the essay explores useful strategies to diagnose this phenomenon in the text and the consequences, from a stemmatic point of view, of the diffusion of the particular type of contamination 'of workshop'.
\end{abstract}

La trasmissione orizzontale, se non di un testo, di singole lezioni si confronta [. . .] con una macchia d'olio, che da un punto determinato si allarga a poco a poco sino a coprire tutta una superficie: fino a dove giungerà allargandosi, nessuno può prevedere con sicurezza.

-Pasquali 1988, 141

C

Ome è noto, l'edizione della Commedia rappresenta un lavoro complesso, non solo a causa del testo, sul quale possono nascere mille dubbi e dibattiti, ma anche perché la tradizione dell'opera costituisce uno dei casi più intricati della filologia italiana e romanza.

Essendomi dedicata per piú di dieci anni alla classificazione dei 580 manoscritti superstiti posso dire che, oltre naturalmente al numero elevato di testimoni (per il quale soccorrono i moderni ausili informatici), uno degli ostacoli - e forse l'ostacolo par excellence - di fronte al quale più spesso mi sono trovata è la contaminazione. Non una contaminazione extrastemmatica che vale a recuperare lezioni incognite al resto della tradizione e spesso più "alte" — che può essere un valido ausilio per il filologo alle prese con varianti poco soddisfacenti - ma una contaminazione tra rami della tradizione ben noti, tra famiglie i cui contorni sfumano l'uno 
nell'altro, senza permettere di intravedere una separazione netta, persino tra esemplari singoli (o, meglio, antigrafi). Insomma, pare ci si debba rassegnare a diagnosticare semplicemente una contaminazione sistematica e abbondante, una mescolanza profonda dei modelli testuali, una molteplicità di "contatti laterali" (BeLLONi 2015, 156) in definitiva una dinamica di con-fusione degli antigrafi che costituiva un perno dei meccanismi di copia manoscritta dell'opera in Toscana.

Se dal punto di vista della classificazione stemmatica non si giunge quindi a un risultato troppo brillante - si potranno al massimo riconoscere le fonti di prelievo più vistose nella confezione di un manufatto contaminato e scartare questi esemplari come inutiles -, dal punto di vista della metodologia critica la tradizione della Commedia si presta a rappresentare un impareggiabile campo di prova per sviluppare riflessioni e teorie di portata generale.

Nel prosieguo di questo intervento affronterò quindi tre ordini di considerazioni, che scaturiscono dall'esame di casi specifici di contaminazione riscontrabili nel grande bacino dei testimoni della Commedia.

\section{$\S$}

1. Partiamo dalla definizione stessa di contaminazione. In molti pregevoli manuali e interventi su questo tema, il fenomeno della contaminazione viene ricondotto al concetto di editio variorum, che è il risultato dell'azione di collazioni multiple, differite e spesso ad opera di diversi soggetti su un esemplare.

Ad esempio, Stussi spiega così la contaminazione:

il fenomeno si verifica di frequente nei centri scrittori di qualche importanza dove erano disponibili più codici contenenti la stessa opera dai quali attingevano i copisti per migliorare o correggere il testo in singoli punti; c'è ragione di ritenere che talvolta a tale scopo fosse allestito un collettore di varianti (editio variorum) cioè un manoscritto dove, sui margini o nell'interlinea, si trovavano annotate le diverse lezioni presenti in altri manoscritti.

$(2005,135)$

Similmente la Ageno:

Accadeva non di rado che non solo un manoscritto contenente un testo greco o latino, ma anche un codice contenente un testo romanzo 
venisse collazionato con un manoscritto di diversa tradizione, e un revisore, attenendosi al nuovo manoscritto, riempisse le lacune, correggesse gli errori evidenti o quelli che lui sembravano tali, segnasse in margine la possibilità di leggere diversamente una parola una frase (in genere, facendo precedere la variante dal segno al 'alias'). Quando il manoscritto che aveva subito la revisione diventava a sua volta modello per altre trascrizioni, il copista poteva "scegliere" alternativamente la lezione del testo o la variante segnata in margine: nasceva così un codice il cui testo era "contaminato".

$(1999,73)$

Credo che troppo spesso si faccia confusione tra contaminazione fisica tra esemplari e contaminazione testuale. Mi spiego subito. Un processo riguarda il prelievo da uno o più codici, oltre a quello di base, da cui si copia il testo, di lezioni giudicate potiori, interessanti, in definitiva migliori consegnate a un supporto materiale, sul quale si trovano a convivere. Da questo processo nasce dunque un prodotto tangibile riconoscibile, un manoscritto con lezioni a margine, in interlinea, con una sua fisionomia precisa e con uno scopo che può essere editoriale, esegetico o di profonda e consapevole curiosità intellettuale. Il secondo riguarda la mescolanza involontaria che si genera, precipuamente in bottega, quando nel testo vengono a confluire diversi rivoli della tradizione, alterandone la fisionomia. Il prodotto di questo secondo procedimento è un testo alterato, contenuto in un manoscritto che può anche avere una veste grafica pulita, nitida, con margini e interrighi liberi e senza stacchi meccanici (di penna, di formato, codicologici). ${ }^{1}$

Si tratta insomma di due entità molto diverse, che in concreto possono rappresentare due fasi interconnesse, ma che in realtà costituiscono due fenomeni intrinsecamente distinti. Il fine della editio variorum è infatti la conservazione di più varianti, la discussione delle lezioni concorrenti, la compresenza in un unico esemplare di varie e differenti alternative entro cui scegliere, entro cui promuovere un dibattito, entro cui verificare le molteplici espressioni della lingua, della prosodia e della cultura. Il telos dell'atto di contaminazione di bottega è, invece, quasi esclusivamente la rapidità nella confezione della copia e il controllo della qualità del testo; l'epurazione dagli errori che avveniva ad opera di copisti incaricati o capibottega che verificavano, attraverso un codice reputato fededegno, che non fossero presenti sviste.

1. Su questi aspetti si veda VARVAro 2010. 
Insomma, esiste una contaminazione materiale, di cui resta traccia, e che è incarnata da quell'esemplare in cui convivono lezioni attinte da diversi modelli, il noto LauSC ad esempio, ${ }^{2}$ ed esiste una contaminazione occulta che trova espressione nei tanti codici contaminati che escono dalle botteghe e che rilevano non in quanto singole copie, ma in quanto massa di esponenti di una o più vulgate, i manoscritti parm $\mathcal{E}$, la tradizione vaticana e Boccaccio ecc. ${ }^{3}$ Oramai sappiamo che in queste botteghe, all'esplodere della produzione in serie tosco-fiorentina, ma già in parte anche a Bologna, la copia avveniva con modalità affini a quelle della pecia. Negli atelier scrittori, le Commedie modello, afferenti a diversi tipi testuali, si presentavano sfascicolate (divise in fascicoli appunto o in cantiche); le unità di copia venivano quindi copiate e poi sottoposte a controlli testuali e a correzioni da parte di copisti-revisori e solo in fine assemblate, arbitrariamente, in una fase ulteriore.

L'osservazione della tradizione superstite ci conforta in queste osservazioni. Il LauSC, l'editio variorum approntata da Villani per svolgere le sue pubbliche letture dantesche, è un codice per cui non si riscontra una discendenza numerosa. Tutt'altro, a patto di considerare il "prodotto finito", ossia la veste con cui si presenta a seguito degli interventi di correzione e marginali, si possono individuare un affine molto stretto, anzi una copia pedissequa, Nap. XIII C 3, che ne assorbe le lezioni a testo e a margine (MANFREDi 1994-1995), e altri sette codici riuniti nella famiglia berlcaetsc, due dei quali, Caet e Laur. 90 sup. 132, sono di mano dello stesso copista: Luigi di Ser Michaelis. Si tratta di un insieme di testimoni che risalgono a iniziative editoriali colte avviate nella Firenze del Salutati per le quali il pregio della compresenza di più tradizioni in un unico supporto rappresenta un valore da mantenere più che un'opportunità di con-fusione. ${ }^{4}$

Un buon esempio della contropartita è la proliferazione, massiccia e in una certa misura irriducibile a una qualunque classificazione ordinata, dei codici parmÉ. Si tratta dei codici riconducibili alle collaborazioni tra i

2. Il manoscritto, copiato da Filippo Villani alla fine del 1300, è quasi certamente la copia approntata per condurre la sua Expositio seu Comentum super 'Comedia' Dantis Allegherii e le letture pubbliche fiorentine, precedentemente tenute da Boccaccio. Il codice presenta numerose varianti al margine e in interlinea, e alcune rasure. Tra le sue fonti si individua con certezza un modello vatbocc (= i codici che si raccolgono attorno all'officina del copista di Vat e alle copie di Boccaccio), accanto ad uno di tipo a0 (= il gruppo di mss. affini di $a$, Mart Triv).

3. Per la definizione di questi gruppi mi permetto di rinviare a Tonello 2018, 105-222; 363-416.

4. Rimando nuovamente a Tonello 2018, 147-57. 
copisti di Parm, per l'appunto, di Ashb, di Ricc. 1025, di Fior. II I 30 ecc., studiati approfonditamente da Pomaro, la quale ha dimostrando che, sui prodotti di questi scribi, gli interventi di revisione, parziali copiature estemporanee, rubriche e così via erano affidati ai capomastri che utilizzavano un proprio antigrafo personale, ovvero un modello testuale preciso (Ром ARo 1994). A questo fenomeno, che potrebbe leggersi come contaminazione di lezioni, si aggiunge quello della contaminazione per giustapposizione, generato dalla mescolanza delle unità di copia, fascicoli e cantiche, separatamente prodotte da più scribi. Ho chiamato altrove questa combinazione "contaminazione ibrida" (Tonello 2016 e 2018, 141-43 e passim); ritengo che si tratti della modalità più diffusa di contaminazione, senza dubbio per la Commedia, ma anche in altre tradizioni, e che, come ho detto, differisca sostanzialmente negli scopi e negli esiti dalla operazione di contaminazione per l'approntamento dell'editio variorum.

2. Passiamo ad un altro ordine di osservazioni. Esistono alcune condizioni che soccorrono la diagnosi di contaminazione di un codice. Si tratta, in prima battuta, della presenza di doppie lezioni, di particolari explicit che dichiarino gli intenti del copista, e della presenza del commento, per via di quello che Procaccioli ha felicemente definito "strabismo operante" (Procaccioli 2001, 77) tra testo al centro della pagina e esegesi a lato, che spesso porta a modificare i versi del poema sulla base dei lemmi del commento, fondati su un testo evidentemente diverso da quello che si sta copiando. In secondo luogo, un utile conferma ex post può essere la presenza di errori patenti, marchiani. Infatti, in contesto di contaminazione, può essere molto istruttivo osservare gli errori patenti, che spesso sono la spia di una evidente azione rielaborativa del copista. Propongo anche in questo caso un esempio concreto: Chig. L IV 109 presenta errori come: 1.28 Poi ch'ei posato un poco il corpo lasso P] mi prese lena e 1.9.53 dicevan tutte riguardando in giuso $\mathrm{P}]$ tenendo tutte il viso. Sono, oltre che singulares, errori così gravi che snaturano la fisionomia del verso rivelando un allontanamento dal testo di partenza molto profondo.

Verosimilmente, la catena di errori che si è prodotta, ovvero il numero di interpositi che si sono succeduti, ha provocato una alterazione del testo tale da rendere possibile il generarsi di tali errori. Laddove ci si trovi di fronte a un certo numero di questi errori patenti si può essere, di fatto, inibiti nella classificazione dal momento che la loro presenza sottrae spazio alle innovazioni congiuntive. Ciò non costituisce un problema nei casi di discendenza 
verticale e di tradizione inerte, per cui di solito l'errore patente si genera per cattiva lettura o, più comunemente, per colmare lacune meccaniche. Si riscontrano infatti, di norma, ancora sufficienti errori congiuntivi grazie ai quali la classificazione è comunque possibile.

Ma statisticamente risultano molti di più i casi in cui gli errori patenti si riscontrano nei manoscritti contaminati. Non solo, infatti, in codici molto contaminati, se ne rileva una presenza massiccia, ma in questo stato di cose essi sono davvero in grado di impedire la classificazione. Si sostituiscono infatti alle già scarse e instabili prove di discendenza. Non bisogna però pensare che siano dovute solo al moltiplicarsi degli atti di copia. Ricordo in proposito il punto 6 del celebre decalogo sul problema della contaminazione di Segre: "si può dire che, mentre la contaminazione sporadica corrisponde a un intento di fedeltà, la contaminazione fitta o multipla suggerisce un senso di relatività, invita a raggiungere, con mezzi autonomi, una almeno speciosa scorrevolezza: il copista si fa, di cercatore, creatore di varianti" (SEgRe 1961, 65). La ragione non è difficile da intuire e risiede nel nesso che lega i copisti per passione alla contaminazione di lezioni. Diversamente dalla contaminazione di bottega che è, come abbiamo detto, per sua natura per giustapposizione - e incidentalmente anche di lezioni -'abitudine dei copisti per passione ad intervenire sul testo, che si concretizza sia andando a caccia della migliore lezione, sia congetturando, finisce per creare esemplari sfigurati, tanto dal punto di vista della discendenza che da quello della corruzione testuale. ${ }^{5}$

Tuttavia, se non m'inganno, stanti così le cose, le serie di errori patenti possono essere recuperate e valorizzate come utili conferme alla classificazione, a patto di interpretarle come indizi confermativi di una avvenuta contaminazione. D'altronde, come afferma Segre, "esiste un legame tra contaminazione e rimaneggiamento" $(1996,65)$.

3. In ultimo, è opinione comune che questo tipo di manoscritti rappresenti un anello terminale della catena generativa della copia, un ultimo tassello che può agevolmente essere tralasciato per la ricostruzione testuale. Ma si dà il caso che i codici contaminati di cui abbiamo parlato, i codici di bottega, siano altamente fecondi, per loro propria natura. E da ciò deriva la difficoltà di riconoscerli, come verrebbe immediatamente da pensare, quali

5. Rimando ancora a Segre 1961, specie per i punti 1-5 e a VARVARo 2010. 
inutiles, proprio per il peso che finiscono per rappresentare nella classificazione.

È evidente invece come, in particolare nelle tradizioni sovrabbondanti, i casi di contaminazione possiedano una capacità produttiva. Nella Commedia è infatti possibile toccare con mano il processo di generazione di tradizioni autonome a discendenza verticale a partire dalla fissazione di testi contaminati. Fornisco anche in questo caso un esempio. Osserviamo il codice Cors. Ross. 368. Il manoscritto, della seconda metà del XIV sec., sottoscritto da Giovanni da Parma, probabilmente un frate, reca segni evidenti di contaminazione: doppie lezioni, varianti nel margine e in interlinea, rasure e correzioni. In Inferno e Purgatorio presenta lezioni provenienti da gruppi molto estesi: d' (D5 D6 D10 D13 D15); vatbocc (*B5, VB9 [che], *V14, V22) e qualche traccia dall'area del cento: PR21 e, in doppia lezione, C28 (2.23.5 vienne oramai, ché 'l tempo che n’è imposto] ogi omai). ${ }^{6}$ Inoltre lezioni singolari: 1.4 .83 vidi quattro grand'ombre $a$ noi venire P] [grand'] contro; 1.5.34 quando giungon davanti alla rovinal di venti; 2.2 .44 tal che faria beato pur descripto P] parea - per soscripto; 2.32.147 simile mostro visto ancor non fue] in vostra vista). Infine, presenta affinità con altri due codici, anch'essi di chiara indole contaminatoria: Vat. 4777' (XV sec. in.) e Ver. Com. 2856' (XIV ex.-XV in.). Ora con entrambi, ora con uno solo dei due. Questi, a loro volta, mostrano un ventaglio di lezioni di diversa provenienza, spesso in comune (Ver. Com. 2856: *B5, D4, *V8, *V14 / C15, L2, D5, D7, D9, D10, D11, D13, D15, D18, D19; Vat. 4777’: D1, *B5, VB9 / C15, D5, D6, C19, D9, D10, D11, D12, D13, D14, D15, D17, D18), e poi ognuno lezioni singolari (2.4.72 che mal non seppe carreggiar Fetòn P] per mal Ver. Com. 2856; 2.8.53 giudice Nin gentil, quanto mi piacque P] giuditio in vie Ver. Com. 2856'; 1.20.80 Ne la qual si distende e la 'mpaluda P] Nel — et la padularda Vat. 4777'; 2.9.42 come fa l'uom che, spaventato, agghiaccia P] spaventando a caccia Vat. 4777').

Questi ultimi due codici, Ver. Com. 2856' e Vat. 4777', oltre alle lezioni esclusive in comune con Cors. Ross. 368' e alle innovazioni loro proprie, presentano una serie di accordi tra loro, a cui si sottrae Cors. Ross. 368'. Se

6. Impiego qui il sistema di riferimento sintetico messo a punto in Tonello 2018. Ho infatti individuato le innovazioni tipiche delle maggiori famiglie del tosco-fiorentino $\alpha$ e ad ognuna ho assegnato un rimando numerato. Per la famiglia d', che comprende le grandi famiglie vatbocc, parmE e cento, faccio riferimento alla tav. 62 , p. 232-35. Per tutte le altre sottofamiglie si rimanda agli elenchi alle pp. 41-51 (ad es. le innovazioni di $a$ ' saranno compendiate come A1 $=1.1 .47$ con la testa alta e con rabbiosa fame] bramosa $a^{\prime}$; A2 $=1.2 .56$ e cominciommi a dir soave e piana] [e] — parlar $a^{\prime}$ ecc.). 


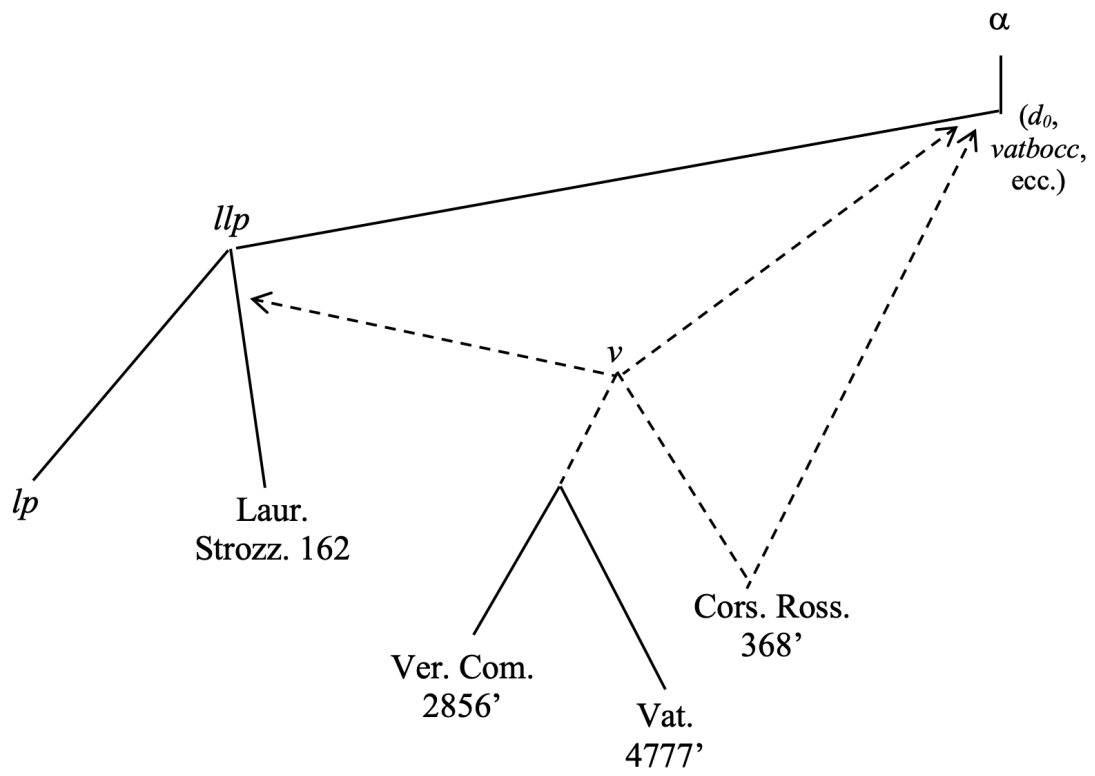

Figura 1. Stemma di $v$ in Inferno e Purgatorio.

ne concluderà che, a monte di Cors. Ross. 368' Vat. 4777' Ver. Com. 2856' vi doveva essere un antigrafo comune, che nominerò $v$, (magari in veste editio variorum) cui può aver attinto Cors. Ross. $368^{\prime}{ }^{7}$ Ma non è finita qui. Spesso alle innovazioni esclusive di $v$ si trova allineato Laur. Strozz. 162, un codice appartenente a una sottofamiglia che fa capo al noto ms. Lau, dalla fisionomia piuttosto indipendente. Da un lato sembrerebbe occupare una posizione più alta rispetto ai suoi consanguinei, sottraendosi spesso all'errore, dall'altro lato compie alcuni errori di distrazione e banalizzazioni, a partire dalla lezione buona o dalla lezione tipica della famiglia lauÉ, che dunque potrebbero essere facilmente ricondotti all'iniziativa del copista.

Ad ogni modo, il contributo testuale di Laur. Strozz. 162 (o un suo affine) nei confronti di Cors. Ross. 368' Vat. 4777' Ver. Com. 2856' è indubitabile. Ecco una rappresentazione stemmatica dei rapporti tra questi codici per Inferno e Purgatorio.

7. L'ipotesi alternativa, pure possibile, che solo Vat. 4777' e Ver. Com. 2856' derivino da uno snodo comune e rappresentino una fonte testuale alla quale Cors. Ross. 368' attinge è di fatto indifferente (e insondabile) rispetto all'altra dal punto di vista dei risultati ottenuti. 
Considerando la poca parte di testimoni giunti fino a noi rispetto all'albero reale non siamo di fatto in grado di ricostruire i passaggi che portano alla nascita di nuove tradizioni. Tuttavia, attraverso casi come questo, possiamo osservare, seppur solo a spanne, come l'intersezione e la mescolanza di tipi testuali possa dar vita a snodi stabili, come dimostra l'uso, imprescindibile in casi come questo, della linea continua. Insomma, i codici affetti da contaminazione non solo non sono isolati e dunque sterili, ma rappresentano un valido spaccato per osservare i processi generativi più complessi della copia manoscritta. Insomma, anche se "contro la contaminazione non si è ancora scoperto alcun rimedio" come recita il citatissimo monito di Paul Maas, (MA As 1972, 62) si dovrà tuttavia convenire che la metodologia con la quale la si affronta può risentire della conoscenza fenomenologica di queste pratiche e che le nostre esperienze critiche possono contribuire al dibattito su questo fondamentale ostacolo della critica stemmatica.

Università Telematica E-CAMPUS

\section{Bibliografia}

\section{Indice dei manoscritti e gruppi citati}

Ashb $=$ Firenze, Biblioteca Medicea Laurenziana, Ashburnhamiani 829

Berl = Berlin, Staatsbibliothek Preußischer Kulturbesitz, Ital. 136

Caet $=$ Rom A, Biblioteca della Fondazione Camillo Caetani, Caetani

Chig. L IV 109 = Città del Vaticano, Biblioteca Apostolica Vaticana, Chigiani L IV 109

Cors. Ross. 368 = Rom A, Biblioteca dell'Accademia dei Lincei e Corsiniana, 44 F 31 (Rossi 368)

Fior. II I 30 = Firenze, Biblioteca Nazionale Centrale, Fondo Nazionale II I 30

Lau $=$ Firenze, Biblioteca Medicea Laurenziana, Pluteo 4016

Laur. 90 sup. 132 = Firenze, Biblioteca Medicea Laurenziana, Pluteo 90 sup. 132

Laur. Strozz. 162 = FIRENZE, Biblioteca Medicea Laurenziana, Strozziani 162

LauSC $=$ Firenze, Biblioteca Medicea Laurenziana, Plutei 26 sin. I

Lo = Belluno, Biblioteca del Seminario, 35

Mart $=$ Milano, Biblioteca Nazionale Braidense, Aldina AP xvi 25

Nap. XIII C 3 = NA POLI, Biblioteca Nazionale "Vittorio Emanuele III", XIII C 3

Parm = PARm A, Biblioteca Palatina, Parmense 3285

Ricc $=$ FIRENZE, Biblioteca Riccardiana, 1010

Ricc. 1025 = FIRenze, Biblioteca Riccardiana, 1025

Triv $=$ Milano, Biblioteca dell'Archivio Storico Civico e Trivulziana, 1055

$\mathrm{T} z=$ Milano, Biblioteca dell'Archivio Storico Civico e Trivulziana, 1077

Vat = Città del Vaticano, Biblioteca Apostolica Vaticana, Vaticani latini 3199 
Vat. 4777 = Città del VAticano, Biblioteca Apostolica Vaticana, Vaticani latini 4777

Ver. Com. 2856 = Verona, Biblioteca Comunale, 2856

$a 0=$ Mart Triv e affini

berlcaetsc $=$ Berl Caet LauSC e affini

bocc $=$ To Ri Chig e affini

cento $=$ Lau Lo Ricc Tz e affini

$\mathrm{d} 0$ = cento parmEु vatbocc

lauE్ = Lau e affini

parm $\mathscr{E}=$ Parm e affini

vat $=$ Vat e affini

vatbocc $=$ vat bocc e affini

\section{Bibliografia}

Ageno, Franca Brambilla. 1999. L'edizione critica dei testi volgari. Padova: Antenore. Belloni, Gino. 2015. "Contaminazione in filologia". Acta Histriae 23: 153-68.

MaAs, Paul. 1972. Critica del testo, translated by Nello Martinelli, presentation by Giorgio Pasquali. Firenze: Le Monnier.

Manfredi, Marinella. 1994-1995. La Commedia secondo il ms. Nap. XIII C 3, tesi di laurea, tutor Federico SANGUineti. Facoltà di Lettere e filosofia, Università degli studi di Salerno.

Pasquali, Giorgio. 1988. Storia della tradizione e critica del testo. Firenze: Le Lettere.

Peтrocchi, Giorgio, ed. 1966-1967. Dante Alighieri, La Commedia secondo l'antica vulgata. Milano: Mondadori.

Pomaro, Gabriella. 1994. Frammenti di un discorso dantesco. Modena: Comune di Nonantola-Poligrafico Mucchi.

Procaccioli, Paolo, ed. 2001. Cristoforo Landino, Comento sopra la Comedia, I-III. Roma: Salerno Editrice.

Segre, Cesare. 1961. "Appunti sul problema delle contaminazioni nei testi in prosa". In Studi e problemi di critica testuale, 63-7. Bologna: Commissione per i testi di lingua.

Stussi, Alfredo. 2005. Introduzione agli studi di filologia italiana. Bologna: Mulino.

Tonello, Elisabetta. 2016. "La contaminación en la Commedia". Creneida. Anuario de Literaturas Hispánicas 4.

- 2018. Sulla tradizione tosco-fiorentina della Commedia di Dante (secoli XIV-XV). Padova: libreriauniversitaria.it.

VArvaro, Alberto. 2010. "Considerazioni sulla contaminazione, sulle varianti adiafore e sullo stemma codicum". In Storia della lingua italiana e filologia, Atti del VII Convegno ASLI, Associazione per la Storia della lingua italiana (Pisa-Firenze, 18-20 dicembre 2008), edited by Claudio Ciociola, 191-96. Firenze: Cesati. 